\title{
EFFECTS OF SOLVENT POLARITY ON SOLVATION FREE ENERGY, DIPOLE MOMENT, POLARIZABILITY, HYPERPOLARIZABILITY AND MOLECULAR REACTIVITY OF ASPIRIN
}

\author{
MOHAMMAD FIROZ KHAN ${ }^{1}$, RIDWAN BIN RASHID ${ }^{1}$, MUHAMMED MAHFUZUR RAHMAN ${ }^{1}$, MD. AL FARUK ${ }^{2}$, \\ MD. MUSTAFEZUR RAHMAN ${ }^{2}$, MOHAMMAD A. RASHID ${ }^{3 *}$
}

1Department of Pharmacy, State University of Bangladesh, Dhaka-1205, Bangladesh, 2Department of Pharmacy, Daffodil International University, Dhaka, Bangladesh, ${ }^{3}$ Department of Pharmaceutical Chemistry, University of Dhaka, Dhaka 1000, Bangladesh Email: ridwan@sub.edu.bd

Received: 24 Oct 2016 Revised and Accepted: 21 Dec 2016

\section{ABSTRACT}

Objective: The aim of the study is to explore the effects of solvent polarity on solvation free energy, dipole moment, polarizability, first order hyperpolarizability and different molecular properties like chemical hardness and softness, chemical potential, electronegativity, electrophilicity index of aspirin which may lead to better understand the reactivity and stability of aspirin in different solvent systems.

Methods: Becke, 3-parameter, Lee-Yang-Parr (B3LYP) level of theory with 6-31G(d,p) basis set was employed to conduct all type of calculations for both in the gas phase and in solution. The solvation free energy, dipole moment and molecular properties were calculated by applying the Solvation Model on Density (SMD) in four solvent systems namely water, methanol, ethanol and $n$-octanol.

Results: The solvation energies steadily increased as the dielectric constant was decreased i.e. free energy increases with decreasing polarity of the solvent. The dipole moment of aspirin was found to be increased when going from non-polar to polar solvents. The dipole moment of aspirin was higher in different solvents than that of the gas phase. The polarizability and first order hyperpolarizability were also increased with the increasing dielectric constant of the solvent. Moreover, ongoing from non-polar to polar solvent the chemical potential, electronegativity and electrophilicity index were increased except in $n$-octanol. The chemical potential, electronegativity and electrophilicity index of aspirin in $n$-octanol was higher than that of ethanol. On the other hand, chemical hardness was increased with decreasing polarity of the solvent and the inverse relation was found in the case of softness.

Conclusion: The calculated solvation free energy, dipole moment, polarizability, first order hyperpolarizability and molecular properties found in this study may lead to the understanding of stability and reactivity of aspirin in different solvent systems.

Keywords: Aspirin, Solvation free energy, Dipole moment, Solvation model, Polarizibility

(C) 2017 The Authors. Published by Innovare Academic Sciences Pvt Ltd. This is an open access article under the CC BY license (http://creativecommons.org/licenses/by/4. 0/) DOI: http://dx.doi.org/10.22159/ijpps.2017v9i2.15853

\section{INTRODUCTION}

Non-steroidal anti-inflammatory drugs (NSAIDs) are a class of compounds that block cyclooxygenase (COX) enzyme involved in the first step of the arachidonic acid cascade. COX exists in two isoforms namely COX-1 and COX-2. The first is constitutively expressed in stomach, kidneys and platelets and is considered important in mucosal protection and platelet function. COX-2 is inducible and plays a major role in prostaglandin biosynthesis in inflammatory cells [1].

Aspirin (fig. 1) is a prototype NSAID and is used to treat pain, fever, and inflammation. Aspirin also inhibits the platelets aggregation. It is a non-selective COX-2 inhibitor and inhibits both the isoforms of COX enzyme. The therapeutic effects of aspirin are obtained due to the inhibition of COX-2; on the other hand, inhibition of COX-1 leads to undesirable side effects on the gastrointestinal tract such as ulceration, bleeding and perforation of the gastrointestinal tract.

Low dose of aspirin is effective in preventing heart attacks, strokes and blood coagulation [2]. Besides, low doses of aspirin are also administered to a patient having a heart attack to reduce the risk of another heart attack or death of cardiac tissue [3, 4]. Aspirin is also used to prevent certain types of cancer, particularly colorectal cancer [5].

Several attempts were made to synthesise aspirin derivatives in order to get compounds having desired biological activities with reduced toxicities. Zhen et al. 2014 [6] prepared aspirin derivatives having anti-thrombotic and gastric mucosal protection properties.
Aspirin derivatives having antioxidant, anticoagulant and antiplatelet activities were also reported [7].

Few computational and theoretical studies of aspirin have been reported earlier. El-Shahawy, 2014 reported the theoretical spectral studies of aspirin [8]. Datt et al., 2012 investigated experimental and computational study of the loading and release of aspirin from zeolite HY [9]. Besides, Marjan et al., 2014 conducted a computational study to find the prospect of aspirin side effects [10]. Khan et al., 2015 reported a theoretical study of geometry, molecular properties and molecular docking study of aspirin [11].

The variation in solvent polarity and the type of solute-solvent interaction(s) can affect the geometry, dipole moment, polarizability, hyperpolarizability and other molecular properties [12-14] due to variable interactions with the highest occupied and lowest unoccupied molecular orbitals (HOMO-LUMO) [12, 14, 15]; and hence, can influence the stability and reactivity of the molecule. A detail of the molecular characteristics and interactions can be obtained from the Density Functional Theory (DFT) calculations which eventually lead to a good understanding of molecular properties $[14,16]$.

Hence, as part of our ongoing research $[11,17]$ the present study was undertaken to report the medium effect on solvation free energy, dipole moment, polarizability, first order hyperpolarizability and chemical reactivity of aspirin which would be potentially helpful to better understand the stability of aspirin in different solvent system and for the development of new pharmaceutical and (bio) chemical products derived from aspirin. 
<smiles>CC(=O)Oc1ccccc1C(=O)O</smiles>

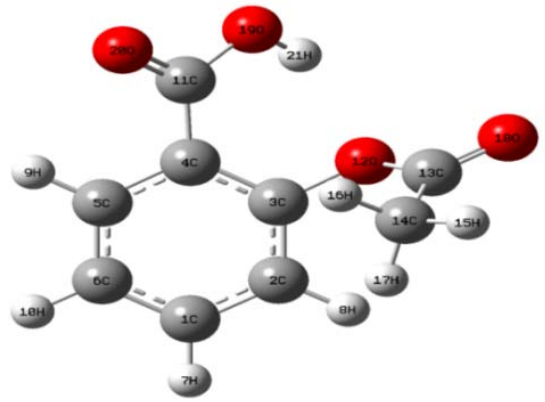

Fig. 1: Structure of aspirin

\section{MATERIALS AND METHODS}

\section{Computational methods}

Gaussian 09 software package [18] was used to perform all type of calculations. From our previous work [11], it was found that geometries of aspirin obtained using the Becke, 3-parameter, Lee-Yang-Parr (B3LYP) with 6-31G(d,p) level of theory showed better agreement with experiments. Therefore, the geometries of aspirin were optimised at the B3LYP level of theory with 6-31G $(\mathrm{d}, \mathrm{p})$ basis set. The optimised geometry was confirmed by the absence of negative frequency in the lowest energy state of the molecule. The solvation free energies, dipole moment and molecular properties were calculated using Solvation Model on Density (SMD) [19] in four solvents such as water, methanol, ethanol and $n$-octanol. All calculations involving solvation were performed using the optimised solution-phase structures.

\section{RESULTS AND DISCUSSION}

\section{Solvation free energy}

The suggested SMD model was used to calculate the solvation free energy in four solvent systems namely water, methanol, ethanol and $n$-octanol. The free energy of solvation $(\Delta G)$ is calculated according to the following equation.

$$
\Delta \mathrm{G}=\mathrm{G}(\mathrm{sol})-\mathrm{G} \text { (gas) }
$$

Where,

$\mathrm{G}($ gas $)=$ Sum of electronic and thermal free energy in gas phase

$\mathrm{G}(\mathrm{sol})=$ Sum of electronic and thermal free energy insolvent

The solvation energies steadily increased in going from higher to lower dielectric constant i.e. free energy increases with decreasing polarity of the solvent (table 1 and fig. 2).

This is due to the different degree of interactions and hence, stabilisation of HOMO-LUMO orbital by the different solvents. From table 4 and fig. 6, it is clear that HOMO-LUMO gap increases with decreasing polarity of the solvents suggesting a higher degree of interactions of aspiring with decreasing polarity of the medium.

However, the differences between computed and experimental free energies [20] in four solvents ranged from 17.41 to $21.90 \mathrm{~kJ} / \mathrm{mol}$.

Table 1: Solvation free energy $(\mathrm{kJ} / \mathrm{mol})$ of aspirin in different solvents with SMD

\begin{tabular}{lll}
\hline Medium (dielectric constant) & B3LYP/6-31G(d,p) & Experimental [20] \\
\hline Water (78.3) & -46.01 & -24.2 \\
Methanol (32.6) & -54.02 & -37.1 \\
Ethanol (24.9) & -54.89 & -37.5 \\
$n$-Octanol (9.9) & -49.24 & -35.5 \\
\hline
\end{tabular}

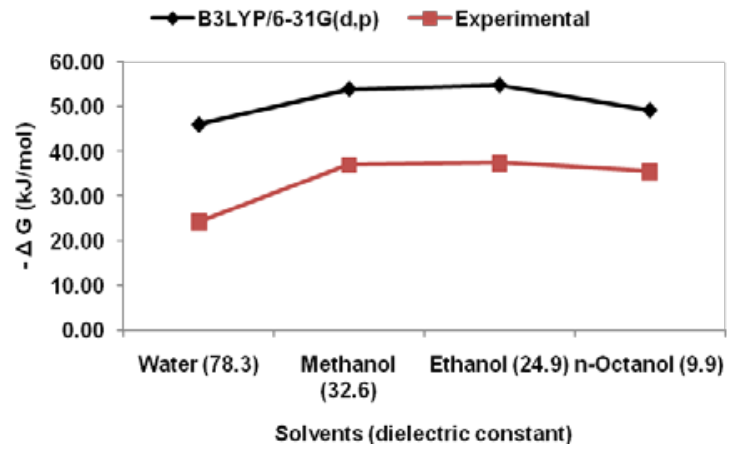

Fig. 2: Effect of solvent polarity on salvation free energy $(\mathrm{kJ} / \mathrm{mol})$ of aspirin

\section{Dipole moment}

The dipole moment is expected to be greater in solution than the corresponding dipole moment in the gas phase. Table 2 presents the dipole moments computed in the gas phase and in different solvents (water, methanol, ethanol and $n$-octanol) at the B3LYP level of theory with $6-31 \mathrm{G}(\mathrm{d}, \mathrm{p})$ as a basis set using SMD solvation model. The dipole moment was gradually increased when going from lower to higher dielectric constant i.e. the dipole moment increases with increasing polarity of the solvent (fig. 3 ).
Table 2: Dipole moment (Debye, (D)) of aspirin in gas phase and in different solvents using SMD

\begin{tabular}{ll}
\hline Medium (dielectric constant) & Dipole moment (D) \\
\hline Gas & 3.8 \\
Water (78.3) & 5.6 \\
Methanol (32.6) & 5.5 \\
Ethanol (24.9) & 5.4 \\
n-Octanol (9.9) & 5.2 \\
\hline
\end{tabular}

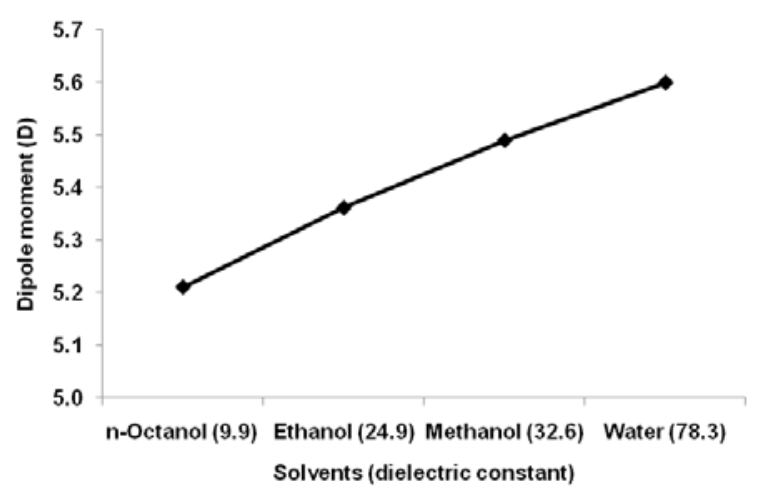

Fig. 3: Effect of solvent polarity on dipole moment (D) of aspirin 


\section{Polarizability and first order hyperpolarizability}

Polarizability is the measure of distortion of a molecule in an electric field. The polarizability $(\alpha)$ was calculated using the following equation:

$$
\alpha=\frac{1}{3}\left(\alpha_{x x}+\alpha_{y y}+\alpha_{z z}\right)
$$

The polarizability is used to determine the strength of molecular interactions and optical properties of a system [21]. A molecule with a low HOMO-LUMO gap is more polarizable and possesses high chemical reactivity, low kinetic stability, and high electro-optic response and is known as soft molecule [21]. The calculated polarizability of aspirin is presented in table 3 and fig. 4 which indicates that the polarizability gradually increases when going from lower higher dielectric constant i.e. the reactivity increases with increasing the polarity of the solvent. This is attributed due to a different degree of interactions of solvents with the HOMO and LUMO orbital of aspirin. Table 4 and fig. 6 indicate that the HOMOLUMO energy gap decreases with increasing dielectric constant of the solvent, thereby the molecule becomes more reactive with increasing polarity of the solvent. However, the polarizability of aspirin in different solvent ranged from 134.90 to $142.91 \mathrm{a}$. u.

The first order hyperpolarizability $(\beta)$ is the measure of the nonlinear optical activity which can be of different types such as $\beta_{\text {vec }}$
( $\beta$ vector), $\beta_{\|}$( $\beta$ parallel $)$ and $\beta_{\text {tot }}(\beta$ total). It is a third rank tensor that can be described by a $3 \times 3 \times 3$ matrix. The 27 components of the 3D matrix can be reduced to 10 components due to the Kleinman symmetry [22]. GAUSSIAN provides 10 components of this matrix as $\beta_{x x x}, \beta_{y x x}, \beta_{x y y}, \beta_{y y y}, \beta_{x x z}, \beta_{x y z}, \beta_{y y z}, \beta_{x x z}, \beta_{y z z}, \beta_{z z z}$, respectively, from which all $\mathrm{x}, \mathrm{y}$ and $\mathrm{z}$ components of $\beta$ can be calculated.

In this investigation, we report $\beta_{\text {tot }}$ for all the solvent systems listed in table 3. The component of $\beta_{\text {tot }}$ can be calculated using the following equation.

$$
\beta_{\text {tot }}=\left(\beta_{\mathrm{x}}^{2}+\beta_{\mathrm{y}}^{2}+\beta_{\mathrm{z}}^{2}\right)^{1 / 2}
$$

Where,

$\beta_{x}=\beta_{x x x}+\beta_{x y y}+\beta_{x z z}$

$\beta_{y}=\beta_{y y y}+\beta_{x x y}+\beta_{y z z}$

$\beta_{z}=\beta_{z z z}+\beta_{x x z}+\beta_{y y z}$

The first order hyperpolarizability was increased when going from lower to higher dielectric constant i.e. the first order hyperpolarizability increases with increasing polarity of the solvent (fig. 5). The difference in hyperpolarizability in different solvents was ranged from 35.95 to $40.06 \mathrm{a}$. u.

\begin{tabular}{|c|c|c|c|c|c|c|c|c|}
\hline Medium (dielectric constant) & $\alpha_{\mathrm{xx}}$ & $\alpha_{\mathrm{yy}}$ & $\alpha_{\mathrm{zz}}$ & $\alpha_{\text {tot }}$ & $\beta_{\mathrm{x}}$ & $\beta_{\mathrm{y}}$ & $\beta_{\mathrm{z}}$ & $\beta_{\text {tot }}$ \\
\hline Gas Phase & 133.03 & 115.95 & 54.74 & 101.24 & 11.00 & 7.25 & 16.83 & 21.37 \\
\hline Water (78.3) & 184.98 & 170.17 & 73.59 & 142.91 & 31.95 & -9.16 & 22.37 & 40.06 \\
\hline Methanol (32.6) & 183.39 & 167.88 & 72.66 & 141.31 & 30.93 & -8.30 & 22.50 & 39.14 \\
\hline Ethanol (24.9) & 181.16 & 165.64 & 71.67 & 139.49 & 29.11 & -7.10 & 22.64 & 37.55 \\
\hline n-Octanol (9.9) & 175.98 & 159.48 & 69.25 & 134.90 & 27.03 & -5.67 & 23.01 & 35.95 \\
\hline
\end{tabular}

Table 3: Medium effect on polarizability (a. u.) and first order hyperpolarizability (a. u.)

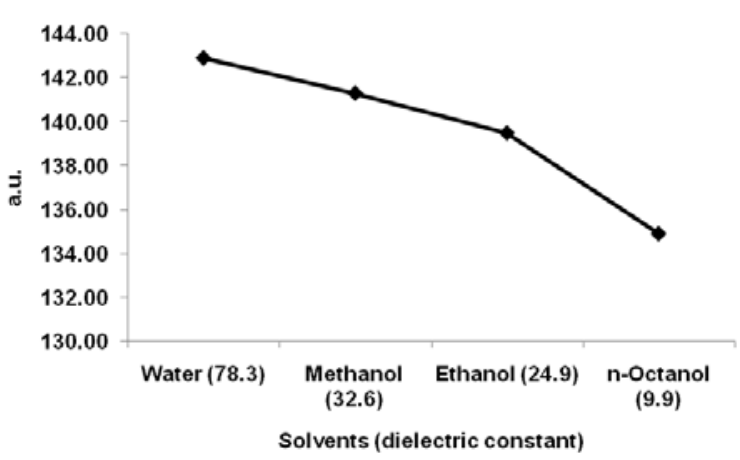

Fig. 4: Effect of solvent polarity on polarizability

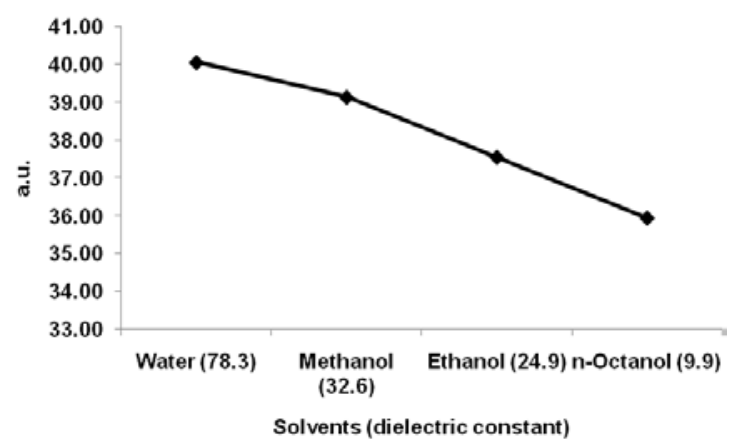

Fig. 5: Effect of solvent polarity on first order hyperpolarizability

\section{Global reactivity descriptors}

The energy gap of HOMO and LUMO determines the molecular electrical transport properties. The HOMO-LUMO energy gap can be used to calculate the global chemical reactivity descriptors of molecules like hardness, chemical potential, softness, electronegativity and electrophilicity index [23-27]. The HOMOLUMO gap of aspirin is presented in table 4 and fig. 6.

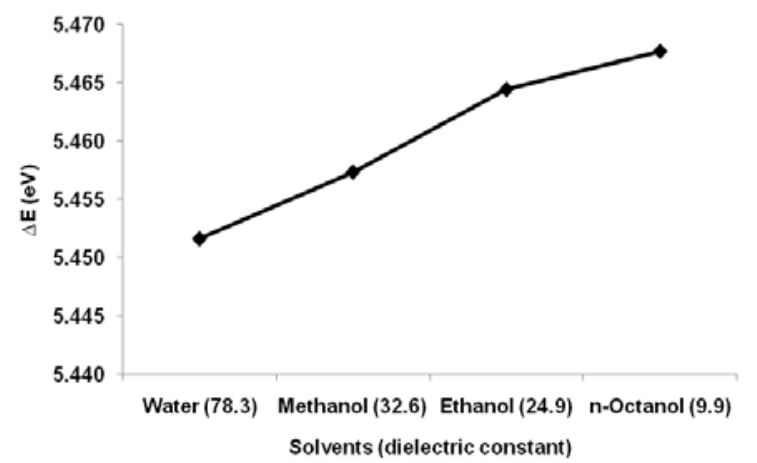

Fig. 6: Effect of solvent polarity on HOMO-LUMO energy gap of aspirin

Koopman's theorem for closed-shell molecules can be applied to calculate the hardness $(\eta)$, chemical potential $(\mu)$ and electronegativity $(\chi)$ and softness $(S)$ by using following formula:

$$
\begin{gathered}
\eta=\frac{I-A}{2} \\
\mu=-\frac{I+A}{2} \\
\chi=\frac{I+A}{2} \\
S=\frac{1}{\eta}
\end{gathered}
$$

Where I and A are the ionisation potential and electron affinity of the molecules, respectively and I =-Еномо, $\mathrm{A}=$--E цимо. 
Molecules with large HOMO-LUMO gaps are known as hard molecule whereas molecules with small HOMO-LUMO gaps are termed as a soft molecule. The stability of the molecule to hardness and softness can be correlated. A molecule with least HOMO-LUMO gap is more reactive and vice versa. Parr et al. 1999 [26] defined the global electrophilic power of a molecule as electrophilicity index $(\omega)$ which can be denoted by the formula as follows:

$$
\omega=\frac{\mu^{2}}{2 \eta}
$$

The above equations are used to calculate chemical potential, hardness and electrophilicity index.
This reactivity quantity has been used to understand the toxicity of various pollutants in terms of their reactivity and site selectivity [28-30]. The molecular properties of aspirin in the gas phase and in the different medium are presented in table 5. Ongoing from non-polar to polar solvent the chemical potential, electronegativity and electrophilicity index were increased except in $n$-octanol (fig. 7 and 8).

The chemical potential, electronegativity and electrophilicity index of aspirin in $n$-octanol was higher than that of ethanol. On the other hand, chemical hardness was increased with decreasing polarity of the solvent and opposite relation was found in the case of softness.

Table 4: Molecular orbital energy (eV) (HOMO and LUMO) of aspirin in different solvents with SMD

\begin{tabular}{llll}
\hline Medium (dielectric constant) & \multicolumn{3}{c}{ Molecular orbital energy (eV) } \\
\cline { 2 - 4 } & HOMO & LUMO & \multicolumn{1}{c}{} \\
\hline Gas Phase & -7.055 & -1.658 & 5.398 \\
Water (78.3) & -6.986 & 5.452 \\
Methanol (32.6) & -6.983 & -1.535 & 5.457 \\
Ethanol (24.9) & -6.956 & -1.525 & 5.464 \\
n-Octanol (9.9) & -6.963 & -1.492 & 5.468 \\
\hline
\end{tabular}

Table 5: Medium effect on molecular properties of aspirin

\begin{tabular}{llllll}
\hline $\begin{array}{l}\text { Medium (dielectric } \\
\text { constant) }\end{array}$ & $\begin{array}{l}\text { Chemical hardness } \\
(\boldsymbol{\eta})\end{array}$ & $\begin{array}{l}\text { Softness } \\
(\mathbf{S})\end{array}$ & $\begin{array}{l}\text { Chemical potential } \\
(\boldsymbol{\mu})\end{array}$ & $\begin{array}{l}\text { Electronegativity } \\
(\boldsymbol{\chi})\end{array}$ & $\begin{array}{l}\text { Electrophilicity index } \\
(\boldsymbol{\omega})\end{array}$ \\
\hline Gas Phase & 2.6988 & 0.3705 & -4.36 & 4.36 & 9.49 \\
Water (78.3) & 2.7258 & 0.3669 & -4.26 & 4.26 \\
Methanol (32.6) & 2.7287 & 0.3665 & -4.25 & 4.25 & 9.08 \\
Ethanol (24.9) & 2.7322 & 0.3660 & -4.22 & 4.22 \\
n-Octanol (9.9) & 2.7339 & 0.3658 & -4.23 & 4.23 \\
\hline
\end{tabular}

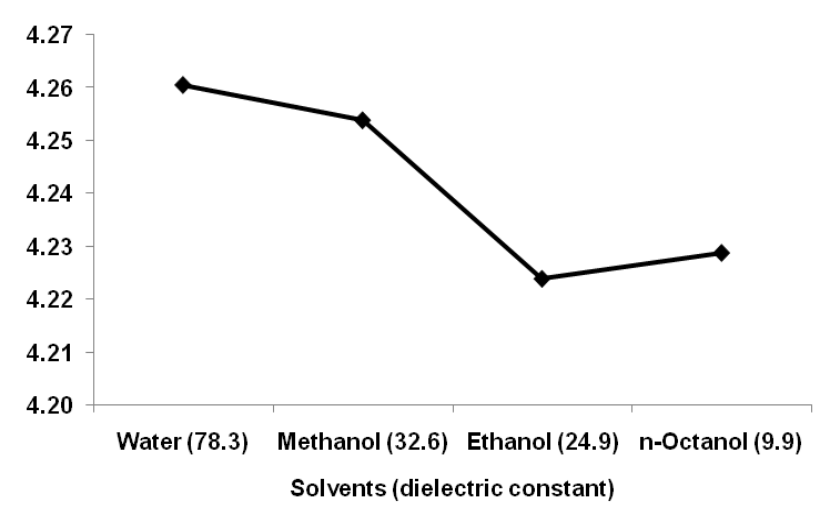

Fig. 7: Effect of solvent polarity on electronegativity of aspirin

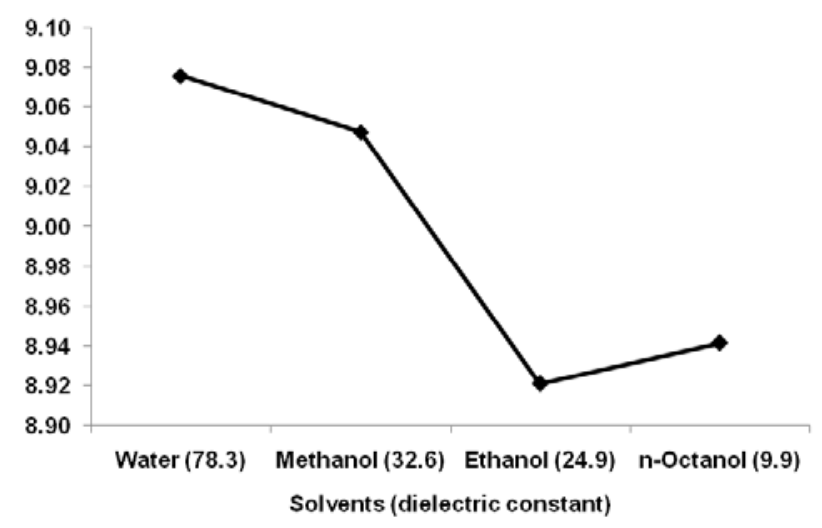

Fig. 8: Effect of solvent polarity on electrophilicity index of aspirin

\section{CONCLUSION}

In the present work, the medium effect on solvation free energies, dipole moment and molecular properties have been determined from B3LYP theory with 6-31G (d, p) basis set. The solvation energies were steadily increased as the dielectric constant was decreased and a similar trend of solvation energy between computational and experimental was found. The dipole moment, polarizability and first order hyperpolarizability of aspirin were gradually increased with the increasing polarity of the solvent. Ongoing from non-polar to polar solvent the chemical potential, electronegativity and electrophilicity index were increased except in $n$-octanol. The chemical potential, electronegativity and electrophilicity index of aspirin in $n$-octanol was higher than that of ethanol. On the other hand, chemical hardness was increased with decreasing polarity of the solvent and the inverse relation was found in the case of softness.

Therefore, it can be concluded that aspirin is more reactive and hence, unstable in a polar solvent which is evident from the polarizability and chemical softness in different solvents. The results obtained in this study may be of assistance in the quest of theoretical evidence for aspirin in reaction intermediates and pharmaceuticals.

\section{CONFLICT OF INTERESTS}

All authors declared that they have no competing interest.

\section{REFERENCES}

1. Griswold DE, Adams L. Constitutive cyclooxygenase (COX-1) and inducible cyclooxygenase (COX-2): Rationale for selective inhibition and progress to date. Med Res Rev 1996;16:181-206.

2. Lewis HD, Davis JW, Archibald DG, Steinke WE, Smitherman TC, Doherty Je JE, et al. Protective effects of aspirin against acute myocardial infarction and death in men with unstable angina. $\mathrm{N}$ Engl J Med 1983;309:396-403.

3. Julian DG, Chamberlain DA, Pocock SJ. A comparison of aspirin and anticoagulation following thrombolysis for myocardial 
infarction (the AFTER study): a multicentre unblinded randomised clinical trial. Br Med J 1996;313:1429-31.

4. Krumholz HM, Radford MJ, Ellerbeck EF, Hennen J, Meehan TP, Petrillo M, et al. Aspirin in the treatment of acute myocardial infarction in elderly medicare beneficiaries. Patterns of use and outcomes. Circulation 1995;92:2841-7.

5. Rothwell PM, Wilson M, Price JF, Belch JFF, Meade TW, Mehta Z. Effect of daily aspirin on risk of cancer metastasis: A study of incident cancers during randomised controlled trials. Lancet 2012;379:1591-601.

6. Zhen X, Zong M, Gao S, Cao Y, Jiang L, Chen S, et al. Preparation and characterization of a novel aspirin derivative with antithrombotic and gastric mucosal protection properties. PLOS One 2014;9:1-9.

7. Cha BC, Lee SB. Synthesis and biological activity of aspirin derivatives. Arch Pharm Res 2000;23:116-20.

8. El-Shahawy A. DFT cancer energy barrier and spectral studies of aspirin, paracetamol and some analogues. Comput Chem 2014;2:6-17.

9. Datt A, Fields D, Larsen SC. An experimental and computational study of the loading and release of aspirin from Zeolite HY. J Phys Chem C 2012;116:21382-90.

10. Marjan MN, Hamzeh MT, Rahman E, Sadeq V. A computational prospect to aspirin side effects: aspirin and COX-1 interaction analysis based on non-synonymous SNPs. Comput Biol Chem 2014;51:57-62.

11. Khan MF, Rashid RB, Rashid MA. Computational study of geometry, molecular properties and docking study of aspirin. World J Pharm Res 2015;4:2702-14.

12. Anbarasan PM, Kumar PS, Geetha M, Govindan R, Manimegalai S, Velmurugan K. Geometries, electronic structures and electronic absorption spectra of silicon dichloride are substituted phthalocyanine for dye-sensitized solar cells. Recent Res Sci Technol 2010;2:8-16.

13. Praveen PL, Ojha DP. Substituent and solvent effects on UVvisible absorption spectra of liquid crystalline disubstituted biphenyl cyclohexane derivatives-a computational approach. Cryst Res Technol 2012;47:91-100.

14. Khan MF, Rashid RB, Islam SM, Rashid MA. Computational study of geometry, solvation free energy, dipole moment, polarizability, hyperpolarizability and molecular properties of 2-methylimidazole. SQU J Sci 2016:21;89-101.

15. Targema M, Obi-Egbedi NO, Adeoye MD. Molecular structure and solvent effects on the dipole moments and polarizabilities of some aniline derivatives. Comput Theor Chem 2013; 1012:47-53

16. Jayaprakash A, Arjunan V, Jose SP, Mohan S. Vibrational and electronic investigations, thermodynamic parameters, HOMO and LUMO analysis on crotonaldehyde by ab initio and DFT methods. Spectrochim Acta Part B 2010;83:411-9.
17. Khan MF, Nabila SA, Rashid RB, Rahman MS, Chowdhury AA, Rashid MA. In silico molecular docking studies of lichen metabolites against the cyclooxygenase-2 enzyme. Bangladesh Pharm J 2015;18:90-6.

18. Frisch MJ, Trucks GW, Schlegel HB, Scuseria GE, Robb MA Cheeseman JR, et al. Gaussian 09, Revision A.02. Gaussian, Inc., Wallingford CT; 2010.

19. Marenich AV, Cramer CJ, Truhlar DG. Universal solvation model based on solute electron density and on a continuum model of the solvent defined by the bulk dielectric constant and atomic surface tensions. J Phys Chem B 2009:113;6378-96.

20. Perlovich GL, Kurkov SV, Andrey N, Kinchin AN, Bauer-Brandl A. Solvation and hydration characteristics of ibuprofen and acetylsalicylic acid. AAPS PharmSci 2004;6:22-30.

21. Targema M, Obi-Egbedi NO, Adeoye MD. Molecular structure and solvent effects on the dipole moments and polarizabilities of some aniline derivatives. Comput Theor Chem 2013; 1012:47-53.

22. Kleinman DA. Nonlinear dielectric polarization in optical media. Phys Rev 1962;126:1977.

23. Parr RG, Donnelly RA, Levy M, Palke WE. Electronegativity: the density functional viewpoint. J Chem Phys 1978;68:380-7.

24. Parr RG, Pearson RG. Absolute hardness: companion parameter to absolute electronegativity. J Am Chem Soc 1983;105:7512-6.

25. Parr RG, Chattraj PK. The principle of maximum hardness. J Am Chem Soc 1991;113:1854-5.

26. Parr RG, Szentpály LV, Liu S. Electrophilicity index. J Am Chem Soc 1999;121:1922-4.

27. Chattaraj PK, Maiti B, Sarkar U. Philicity: a unified treatment of chemical reactivity and selectivity. J Phys Chem A 2003;107:4973-5.

28. Parthasarathi R, Padmanabhan J, Subramanian V, Sarkar U, Maiti B, Chattaraj P. Toxicity analysis of benzidine through chemical reactivity and selectivity profiles: A DFT approach. Int Electron J Mol Des 2003;2:798-813.

29. Parthasarathi R, Padmanabhan J, Subramanian V, Sarkar U, Mait $B$, Chattaraj P. Intermolecular reactivity through the generalised philicity concept. Chem Phys Lett 2004;394:225-30.

30. Parthasarathi R, Padmanabhan J, Subramanian V, Sarkar U, Maiti B, Chattaraj P. Toxicity analysis of 33'44'5-pentachloro biphenyl through chemical reactivity and selectivity profiles. Curr Sci 2004;86:535-42.

\section{How to cite this article}

- Mohammad Firoz Khan, Ridwan Bin Rashid, Muhammed Mahfuzur Rahman, Md Al-Faruk, Md Mustafezur Rahman, Mohammad A Rashid. Effects of solvent polarity on solvation free energy, dipole moment, polarizability, hyperpolarizability and molecular reactivity of aspirin. Int J Pharm Pharm Sci 2017;9(2):217-221. 